\title{
EFICACIA DE PROGRAMAS DE FIDELIZACIÓN EN SUPERMERCADOS. UN ENFOQUE COMPARATIVO DE DOS FORMAS DE CONSEGUIR LA LEALTAD DEL CLIENTE
}

Facultad de Ciencias Económicas y Empresariales-ICADE Universidad Pontificia Comillas de Madrid

Material original autorizado para su primera publicación como artículo en la Revista académica REDMARKA. Revista Digital de Marketing Aplicado

\section{Resumen:}

El concepto de fidelización ha cobrado en los últimos años un gran relieve en todos los sectores empresariales. La distribución comercial minorista no es una excepción, y no pocos estudios constatan que los distribuidores que han alcanzado mayores niveles de fidelidad y retención de sus clientes, también han logrado mayores beneficios y un crecimiento más rápido. Resultados como la repetición de compras y la recomendación de la enseña a otros potenciales clientes constituyen lo que algunos autores han denominado "economías de la lealtad", que permiten reducir los costes de servir a los clientes actuales, así como los de captar nuevos.

No obstante, la proliferación de distintos modelos de programas de fidelización hace necesaria la selección de aquellas técnicas que resulten más satisfactorias para los clientes y más rentables para la empresa.

El objetivo del presente trabajo es analizar las actitudes de los clientes de un supermercado -y determinados comportamientos vinculados a esas actitudes- ante programas de fidelización sustentados en recompensas diferentes.

La metodología del trabajo se concreta en una investigación empírica llevada a cabo a través de una encuesta a clientes titulares de tarjeta de fidelización de una enseña 
española de supermercados que opera a través de dos programas distintos de fidelización.

Como conclusiones del trabajo, se pone de manifiesto una buena capacidad de diferenciación de los elementos clave de cada programa, aunque una escasa notoriedad de las acciones promocionales asociadas a los mismos. Por otra parte, los factores que se muestran como verdaderamente relevantes en la satisfacción con el club de clientes del supermercado son la percepción del valor que ofrece la adscripción al programa de que se trate (sea mediante la compra directa, sea mediante el volumen de compras acumulado), el potencial que presenta la información de las transacciones en punto de venta para adaptar los incentivos a los hábitos de compra $\mathrm{y}$, en última instancia, la satisfacción con el propio establecimiento.

A su vez, se destaca la necesidad de reflexionar sobre la eficacia y la coherencia de la línea estratégica que sigue la compañía en términos de satisfacción de clientes.

\section{Palabras clave:}

Programa de fidelización, valor/recompensa, supermercado.

\section{Abstract:}

LOYALITY CARDS EFFICACY IN SUPERMARKETS. A COMPARATIVE FOCUS OF TWO WAYS TO GET CLIENT'S FIDELITY

In the last years, the term loyalty has become one of the most important tools in all business sectors. The retailing sector is not an exception, and many studies show that dealers who have attained higher levels of loyalty and retention of their customers, have also achieved higher profits and faster growth. Results such as the repetition of purchases and the word-of-mouth to potential customers are what some authors have called "economies of loyalty" that allows to reduce the costs of keeping customers as well as attracting new ones.

However, due to the increased number of loyalty programs, a precise selection of techniques favorable for customers and more profitable for the company as well is required. 
The main purpose of this paper is to analyze the attitudes of customers of a supermarket -and some behaviors linked to those attitudes- who are members of different loyalty programs based on different rewards. The methodology followed is an empirical research conducted through a survey: card holders who participate in two different loyalty programs.

As a result of the survey, it is shown that customer can clearly differentiate the key factors of each program, although all the promotional activities linked to those key factors have a low awareness. On the other hand, items that customers mentioned as very relevant to them are the valuable perception of being members (whether through direct purchases, either through the number of repeated purchases), the importance of getting information from in-store behavior just to tailor incentives to buying habits and the satisfaction with the supermarket. In turn, the survey highlights the need to reflect on the effectiveness and coherence of the strategic line that follows the company in terms of customer satisfaction.

\section{Key words:}

Loyalty cards, value/reward, supermarket 


\section{Planteamiento y estado de la cuestión}

La fidelización de los clientes se ha convertido, hoy en día, en una condición de supervivencia de la empresa, articulando la estrategia de marketing y facilitando la consecución de una ventaja competitiva en el mercado (Martínez-Ribes, et al 1999). En líneas generales, los beneficios que aporta se pueden resumir en:

- Los clientes fieles generan más ingresos que los clientes esporádicos u oportunistas (Baumann, Burton y Elliott, 2005).

- El coste de mantener a los clientes es, normalmente, más bajo que el coste de captación, ya que se simplifican y reducen los costes de venderle y hacerle llegar el producto o servicio adquirido.

- Con una correcta gestión de la fidelización, se incrementa la confianza del cliente y, como resultado de esto, sus compras se incrementan en volumen y valor.

- El cliente fiel por convencimiento (no por cautividad del mercado o por el elevado coste que supone el cambiar de proveedor), es el mejor portavoz de la empresa.

- En muchas ocasiones, la fidelización de clientes se convierte en una barrera de entrada para otros competidores.

- Los clientes fieles son menos sensibles a los precios y perciben los precios elevados en relación con el valor que reciben. Se produce una relación inversa entre la fidelidad y la elasticidad de su demanda.

- El cliente fiel tiende a convertirse en first adopter de los nuevos productos y servicios de la empresa, por lo que se convierte en un "panel" o referencia a la hora de hacer investigación de mercados.

Adentrándonos en el concepto de lealtad -generalmente considerado como sinónimo al de fidelidad-, debemos señalar la existencia dos grandes enfoques en la literatura, en torno a aspectos comportamentales (repetición de compra) o bien 
actitudinales o cognitivos (sentimientos y afectos positivos hacia una entidad marca/servicio/producto/establecimiento-). Mientras que, desde el primer enfoque, los autores se centran exclusivamente en el comportamiento del cliente dirigido a la compra repetitiva de un producto o servicio (Butscher, 1998; Capizzi y Ferguson, 2003; Bennett y Rundle-Thiele, 2005), otras definiciones de lealtad hacen hincapié en la relación entre una actitud determinada frente al producto u organización y, de nuevo, un comportamiento real repetitivo de compra (Dick y Basu, 1994).

Basándonos en este último planteamiento, asumimos un concepto de fidelidad integrado por ambos componentes (actitud y comportamiento) lo que, coherentemente, ha llevado a orientar el presente trabajo al estudio de las actitudes de los clientes -y determinados comportamientos vinculados a esas actitudes- ante estrategias diferentes de recompensa en el contexto programas de fidelización de un mismo supermercado.

Por su parte, la satisfacción de los clientes -como elemento actitudinal- se convierte en un pilar fundamental de la lealtad, además de presentarse como fuente de beneficios para la empresa, en tanto que permite mantener relaciones a largo plazo con sus clientes (Parasuraman, Berry y Zeithaml, 1991; Shani y Chalasani, 1992). Podemos afirmar que la satisfacción o insatisfacción de un cliente viene determinada por la impresión que éste experimenta después de una compra, como resultado de la diferencia existente entre la expectativa (basada en la calidad del servicio contratado o producto adquirido), y la percepción real de la prestación del servicio. En definitiva, el componente más emocional de la gestión de la satisfacción de los clientes está relacionado con el juego de las percepciones y expectativas.

La suma de percepciones y expectativas obliga a las empresas a entender sus servicios desde la perspectiva del valor aportado al consumidor, concepto que se presenta íntimamente ligado al de lealtad en tanto que la búsqueda de valor por el cliente es la causa de su lealtad (Rodríguez, Camarero y Gutiérrez, 2002). Cada vez más empresas interpretan el valor aportado al consumidor con sus productos 0 servicios en clave de experiencias globales satisfactorias de/con los clientes. Este REDMARKA - Revista Académica de Marketing Aplicado - CIECID - Universidad de A Coruña Año I, Número 1, (2009), pp. 205-229 http://www.redmarka.org.ar 
aspecto es el que ha originado conceptos como el de experiential marketing, cuyo objetivo fundamental es la creación de experiencias holísticas en los clientes por medio de marcas o enseñas que lleven asociadas percepciones sensoriales, afectivas y creativas y que les hablen de un estilo de vida (Schmitt, 2000).

Desde la perspectiva de la distribución comercial, el objetivo de la fidelización de clientes se justifica debido al incremento de la competencia entre enseñas y la proliferación de nuevos productos y categorías. Además, la aplicación de programas de fidelización en la distribución y, en particular, en los mercados de gran consumo, adquiere gran importancia cuando las constantes guerras de precios han afectado a la percepción del valor aportado por la enseñas (oferta indiferenciada, productos commodities, etc,).

El fenómeno de los programas de fidelización en la distribución comercial, sustentados en tarjetas de clientes y ligados a distintas recompensas para los socios, ha experimentado un notable crecimiento en los últimos años. Así, en España coexisten más de 40 programas nacionales consolidados, cifra modesta si se compara con los 150 existentes en el Reino Unido, lo que está derivando en un cansancio de los clientes de este tipo de enfoques de la fidelización (Wright y Sparks, 1999). Adicionalmente, la aparición de nuevos planteamientos dentro de los programas de fidelización, cada vez más sofisticados y con un apoyo tecnológico muy importante, son buena prueba de su expansión y consolidación. Por su parte, este hecho motiva que aparezcan nuevos incentivos y estrategias en el sector del retail con los siguientes objetivos (Cuthbertson, 1998; Reinares, 2006):

- Conseguir la fidelidad de los mejores clientes por medio de la implantación de una estrategia relacional que permita incorporar valores de marca a través de los premios.

- Contribuir a la fama y reputación de le empresa por medio de esos clientes a los que se intenta fidelizar.

- Apoyar a los miembros del club y buscar nuevos "adeptos". 
- En los programas multi-sponsor, contribuir al intercambio de clientes entre sectores empresariales que no son competencia.

- Disponer del mayor volumen de información relevante de cada hogar, con el fin de utilizarla para establecer estrategias acordes a los gustos y preferencias de esos hogares.

- Establecer una alta interactividad como base del programa de fidelización., y personalizar todas las comunicaciones.

- Evitar la fuga (churning) de los miembros del programa de fidelización.

- Conseguir que las recompensas obtenidas por los clientes por su fidelidad no terminen en el hecho de la compra, sino que incentiven e incrementen la frecuencia de compra y el ticket medio.

Este tipo de programas no están exento de críticas. La más habitual es que, con frecuencia, responden a una visión de la fidelización como equivalente a unos meros incentivos promocionales, trasladando una herramienta estratégica hacia el terreno táctico, con una visión eminentemente cortoplacista.

Las implicaciones teóricas de los programas de fidelización en el contexto del comercio minorista han sido descritas ampliamente en la literatura nacional e internacional, tanto en el ámbito académico como en el empresarial. Baste mencionar, a modo de ejemplo, los trabajos de autores españoles como Ortega y Recio (1997), Martínez-Ribes et al (1999), Reinares y Ponzoa (2002), Alfaro (2004), Setó (2004) o Alcaide (2006).

Si bien no resulta difícil encontrar referencias que atiendan de forma muy genérica a cuestiones como la utilidad de estos programas, sectores o categorías de producto donde presentan mayor potencial, tipología más apropiada o efecto sobre las transacciones del establecimiento, son más escasos -en particular, en el contexto español- los trabajos que abordan, empíricamente y con profundidad, el tema de la valoración de programas de fidelización. En este sentido merece la pena mencionar el reciente trabajo de Reinares y Reinares (2005). 
Por su parte, una interesante aportación de este trabajo se deriva de aunar el análisis de la satisfacción con el programa de fidelización y de la satisfacción con el propio establecimiento, dimensiones que se presentan habitualmente disociadas en la literatura.

\section{Objetivos y diseño de la investigación}

La investigación tiene por objetivo, en primer lugar, el análisis de las actitudes de los clientes hacia programas de fidelización con diferentes estrategias para abordar, en segundo término, el análisis del comportamiento de compra en relación con los programas de fidelización.

El objetivo general planteado se ha concretado, a su vez, en cuatro objetivos específicos:

1. Analizar la satisfacción con el programa de fidelización

2. Estudiar la actitud ante las acciones promocionales y su notoriedad

3. Estudiar el comportamiento de compra en el supermercado

4. Analizar el uso de la tarjeta del programa, así como la de otros supermercados

Para dar cumplimiento a estos objetivos, nos hemos valido de la infraestructura de la tarjeta del club de clientes de una enseña de supermercados española de ámbito nacional, que opera a través de dos programas distintos de fidelización -cada uno con sus peculiaridades- en función del área geográfica de que se trate. Como representativos de cada tipo de programa hemos seleccionado dos áreas geográficas: Albacete para el Programa "A" y Barcelona para el Programa "B”.

En lo que atañe a las características de cada tipo de programa: el Programa A ofrece acceso a más de 500 artículos con precios especiales señalizados en los lineales. En marca propia el descuento se sitúa en torno al 10\%, y en marcas nacionales, el descuento se sitúa en torno al $7 \%$ en todas las secciones. En 
cualquier caso, el club de clientes dispone de una serie de beneficios comunes a ambos programas: mensaje en $\mathrm{TPV}^{1}$ por compra realizada, venta cruzada en caja, vales descuento en ticket, etc., que también se analizarán en el presente trabajo. El Programa $\mathrm{B}$ se basa en el abono de unos puntos, en función del valor de la compra realizada por el miembro del club, que se convierten en dinero para futuras compras. El único requisito es comprar por un importe mínimo en cada transacción y disponer de un importe mínimo acumulado al cuatrimestre. En la Tabla 1 se detalla la ficha técnica de la investigación.

TABLA 1: Ficha técnica de la investigación

\begin{tabular}{|c|c|c|}
\hline Universo & \multicolumn{2}{|c|}{$\begin{array}{l}\text { Clientes titulares de tarjeta de fidelización del supermercado y, por tanto, adheridos a alguno } \\
\text { de los } 2 \text { programas de fidelización que la enseña gestiona. En la actualidad, el club de } \\
\text { clientes tiene más de } 200.000 \text { socios adheridos. }\end{array}$} \\
\hline Ámbito geográfico & \multicolumn{2}{|l|}{ Albacete y Barcelona } \\
\hline Muestra & \multicolumn{2}{|c|}{$\begin{array}{l}\text { - TAMAÑO DE LA MUESTRA: } 300 \text { individuos de cada programa de fidelización. En total } \\
600 \text { encuestas telefónicas válidas. } \\
\text { - MÉTODO DE MUESTREO: muestreo aleatorio simple } \\
\text { - ERROR MUESTRAL: } \pm 4,08 \% \text { para el caso más desfavorable de } p=q=50 \% \text { y para el total } \\
\text { de la muestra; } \pm 5,77 \% \text { para Cataluña y Albacete de forma independiente, y para } \\
p=q=50 \% \\
\text { - NIVEL DE CONFIANZA: } 95,5 \%\end{array}$} \\
\hline $\begin{array}{l}\text { Técnica de obtención de } \\
\text { información }\end{array}$ & \multicolumn{2}{|c|}{ Encuesta telefónica asistida por ordenador (CATI). } \\
\hline Trabajo de campo & \multicolumn{2}{|l|}{ Enero y febrero 2007} \\
\hline \multirow{2}{*}{ Análisis de la información } & Técnicas estadísticas & Univariables, bivariables y multivariables \\
\hline & Programas informáticos estadísticos & DYANE VERSIÓN 3 \\
\hline
\end{tabular}

\section{Análisis de las actitudes de los clientes hacia los programas de fidelización}

\subsection{Satisfacción con el programa de fidelización}

Tras constatar un nivel alto de satisfacción general de los clientes con el supermercado de la enseña ${ }^{2}$ (media de 8,35 en una escala de 1 a 10 en los clientes del Programa A -correspondiente a la provincia de Albacete- y 8,0 en los clientes del Programa B -Barcelona- y determinar aquellos factores del establecimiento con los

\footnotetext{
${ }^{1}$ Terminal Punto de Venta

2 Obviamente, nos referimos a la enseña cuya información del Club de Clientes sustenta el estudio. No desvelaremos su nombre por cuestiones de confidencialidad.

REDMARKA - Revista Académica de Marketing Aplicado - CIECID - Universidad de A Coruña Año I, Número 1, (2009), pp. 205-229 
que están más satisfechos, se procede a analizar la satisfacción de los clientes con el programa de fidelización.

En primer lugar, puede afirmarse que los clientes están satisfechos con el programa de fidelización del supermercado tal como muestra la puntuación media de 7,51 que le otorgan en una escala de 1 a 10. Existen diferencias en lo que respecta a la satisfacción con los programas en las dos áreas geográficas analizadas: la puntuación en el programa de Albacete es de 7,66 frente al 7,36 en el de Barcelona. De acuerdo con el valor de la $F$ de Snedecor, $F(1,598)=5,1306$, las diferencias entre medias son estadísticamente significativas al nivel del $0,05(p=0,0239)$.

A su vez, se pone de manifiesto que existe correlación entre la satisfacción con el programa y la satisfacción general con el establecimiento. A este respecto, se ha llevado a cabo un análisis de regresión simple entre las dos variables consideradas ( $F$ de Snedecor con 1 y 598 grados de libertad $=147,8962 ; p=0,0000$ ).

Esa satisfacción con los programas también es coherente con la disposición a recomendar el programa declarada por los clientes de las dos áreas analizadas, tal como se refleja en la Tabla 2.

TABLA 2: Disposición a recomendar el programa a familiares o amigos

\begin{tabular}{|l|c|c|c|}
\hline \multicolumn{1}{|c|}{ Recomendación } & Total muestra & \multicolumn{2}{c|}{ Programas áreas geográficas } \\
\cline { 3 - 4 } & $\%$ & A-Albacete (\%) & B-Barcelona (\%) \\
\hline Sólo recomendaría su programa de fidelización & 32,17 & 37,00 & 27,33 \\
\hline $\begin{array}{l}\text { Recomendaría su programa antes que el de otros } \\
\text { supermercados }\end{array}$ & 37,50 & 35,00 & 40,00 \\
\hline $\begin{array}{l}\text { Recomendaría su programa junto con el de otros } \\
\text { supermercados }\end{array}$ & 15,33 & 15,67 & 15,00 \\
\hline $\begin{array}{l}\text { Recomendaría otros programas de otros } \\
\text { supermercados antes que el suyo }\end{array}$ & 9,83 & 11,00 & 8,67 \\
\hline Nunca recomendaría su programa & 5,17 & 1,33 & 9,00 \\
\hline
\end{tabular}


En lo que respecta a los elementos más valorados de cada programa, en ambos casos destacan en los dos primeros puestos, con puntuación por encima del 8, "La forma en que se recompensan sus compras en el supermercado" y "El plazo de caducidad de los cupones descuento que el supermercado entrega" (Gráfica 1).

A continuación, en el caso del Programa A, "El uso de cupones descuento recortables para canjear en el supermercado" y algo por detrás los elementos "Información disponible sobre las ventajas del Club Cliente","Los productos que encuentro con precio especial Club Cliente", "Las ofertas de los vales del resto de productos del Club Cliente (alimentación seca, bebidas, droguería...) y "las ofertas de los vales de productos frescos del Club Cliente (carnicería, pescadería, frutería, ...)". Como aquellos factores menos valorados se presentan "El ahorro obtenido con el importe de los cheques descuento", "La frecuencia con la que recibe vales o tickets en caja" y "Los vales de productos del Club Cliente", este último con una puntuación media por debajo del 7. En el caso del Programa B, las puntuaciones son ligeramente inferiores, con cuatro elementos con puntuación por debajo del 7 .

GRÁFICA 1: Valoración de los elementos de cada programa de fidelización

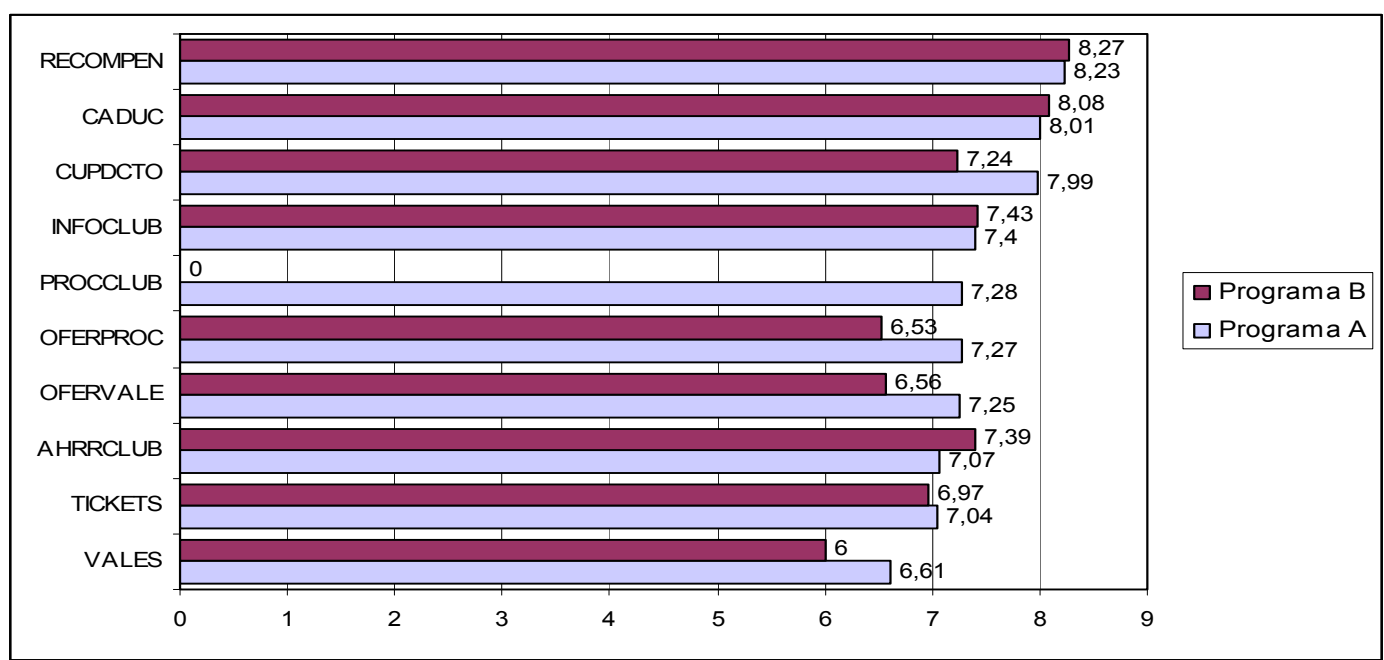


El aspecto diferencial del Programa A, "Los productos que encuentro con precio especial Club Cliente" (PROCCLUB) se sitúa en un término medio, sin presentarse como uno de los más valorados (media $=7,28$ ). Tampoco destaca especialmente en el Programa B su elemento más diferencial, "El ahorro obtenido con el importe de los cheques descuento" (AHORRCLUB, con media $=7,39$ ).

Ahora bien, con el objeto de analizar más en detalle los factores diferenciales de cada programa y dilucidar cuáles de los elementos particulares del programa correlacionan mejor con la valoración global del mismo, se ha realizado un análisis de regresión múltiple tomando como variable dependiente la valoración global del programa de fidelización de que se trate (SATGRAL) y como variables independientes las que se refieren a la valoración de los 10 elementos considerados del programa.

Previamente, y para medir la confiabilidad o consistencia interna de la escala, se ha procedido a aplicar a las variables el test del Coeficiente alfa de Cronbach. El coeficiente $\alpha=0,8016$ indica una considerable consistencia interna.

Como pudo comprobarse a partir de la matriz de coeficientes de correlación simple, la mayor correlación con la valoración global del programa se produce con los elementos "Los productos que encuentro con precio especial Club Cliente" (PROCCLUB) "La frecuencia con la que recibe vales o tickets en caja" (TICKETS) "Las ofertas de los vales de productos frescos del Club Cliente" (OFERVALE) y "Los vales de productos del Club Cliente" (VALES). De acuerdo con el nivel de significación de la $t$ de Student, las variables mencionadas tienen un efecto significativo sobre la variable indicativa de las condiciones globales (las tres primeras al nivel $p<0,01$ ) y la cuarta al nivel $p<0,05$ ).

En el caso del Programa B, se ha procedido de la misma forma: aquellos factores que correlacionan mejor con la valoración global del programa de fidelización son "El ahorro obtenido con el importe de los cheques descuento" (AHRRCLUB), "Los vales 
de productos del Club Cliente" (VALES), "La frecuencia con la que recibe vales o tickets en caja” (TICKETS), y"Las ofertas de los vales de productos frescos del Club Cliente" (OFERVALE).

Los resultados de este análisis subrayan que aquellos elementos que constituyen el principal factor diferencial de cada programa -(PROCCLUB) en el Programa $A$ y (AHRRCLUB) en el Programa B- son los que mejor correlacionan o están más presentes en la valoración global de éste, lo que pone de manifiesto una buena capacidad de diferenciación de los elementos destacados en cada caso.

\subsection{Actitud ante las acciones promocionales y su notoriedad}

A continuación, en relación con el objetivo de analizar la actitud ante las acciones promocionales y su notoriedad, se atiende en primer lugar al recuerdo declarado con relación a las acciones promocionales de cada uno de los programas de fidelización. Ante la pregunta "Si nos centramos en las promociones que nuestro supermercado le envía por correo postal, ¿ ¿recuerda alguna de las últimas que ha recibido?”, puede subrayarse que no llega al $40 \%$ el porcentaje de la muestra que responde afirmativamente. El recuerdo declarado es claramente superior en Programa B: 56\% frente al $23,7 \%$ manifestado en el caso del Programa A (las diferencias son estadísticamente significativas al nivel 0,01: $p=0,0000$ ).

Sin entrar en analizar las diferencias existentes en creatividades, tipos de envío, segmentaciones, etc., parece evidente que el grado de recuerdo en este segundo tipo de programa es mayor. Sin embargo, esta percepción de recuerdo no se corresponde con el corto plazo, ya que el porcentaje de personas que recuerda el último envío, es bastante bajo "¿Recuerda cuál ha sido la última promoción / descuento del que se ha beneficiado en nuestro supermercado?": en media 29,7\%, recuerdo significativamente mayor en el Programa A (35\%) frente a un $24,3 \%$ en el $B(p=0,0042)$. 
Las promociones más interesantes en opinión de los clientes tienen más que ver con el trato y servicios exclusivos, así como los mensajes de ofertas exclusivas (Tabla 3):

TABLA 3: Tipos de promociones que le parecen más interesantes (sugerido)

\begin{tabular}{|l|c|c|c|}
\hline \multirow{2}{*}{ Tipos de promociones preferidas } & \multirow{2}{*}{$\begin{array}{c}\text { Total muestra } \\
\text { \% }\end{array}$} & \multicolumn{2}{c|}{ Programas áreas geográficas } \\
\cline { 3 - 4 } & A-Albacete (\%) & B-Barcelona (\%) \\
\hline $\begin{array}{l}\text { Trato especial (cajas para socios del club, } \\
\text { servicios exclusivos...) }\end{array}$ & 58,33 & 54,33 & 62,33 \\
\hline Información/mensajes de ofertas exclusivas & 20,83 & 27,00 & 14,67 \\
\hline Descuentos en precios & 13,50 & 13,00 & 14,00 \\
\hline Promociones 3x2 & 3,83 & 2,33 & 5,33 \\
\hline Regalos directos & 3,17 & 3,33 & 3,00 \\
\hline Sorteos & 0,33 & 0,00 & 0,67 \\
\hline
\end{tabular}

Base: 600

Ji cuadrado con 7 grados de libertad $=18,2832 \quad(p=0,0108)$

Sobre el recuerdo del último envío realizado, se pregunta de forma sugerida: "Hace un tiempo le enviamos un sobre de color naranja con una promoción que contenía cupones descuento de $2 €$ por cada $20 €$ de compra, ¿lo recuerda?”. Una vez más, se puede observar la baja notoriedad e impacto que ha tenido en los clientes: menos de la mitad de la muestra (48\%) declara recordar la promoción, sin que se muestren diferencias significativas estadísticamente entre ambos tipos de programa.

Pero en el uso final de los cupones descuento parece que haya una tendencia más favorable. En esta ocasión, podemos afirma que los clientes aceptan y usan de forma efectiva las promociones de precio y descuento. Casi el $72,2 \%$ de los clientes redimió los cupones, resultando muy llamativa la diferencia que se produce en el impacto de la promoción en el caso del Programa B, donde un 92,5\% de los clientes hicieron uso de los cupones, frente al $54,6 \%$ que lo hizo en el otro programa ${ }^{3}$.

\footnotetext{
${ }^{3} \mathrm{Ji}$ cuadrado con 1 grados de libertad $=51,5520 \quad(p=0,0000)$

218 REDMARKA - Revista Académica de Marketing Aplicado - CIECID - Universidad de A Coruña Año I, Número 1, (2009), pp. . 237-261

http://www.redmarka.org.ar
} 


\section{Análisis del comportamiento de compra en relación con los programas de fidelización}

\subsection{Comportamiento de compra en el supermercado}

En el área del Programa A, el 69\% de los clientes declara que su establecimiento de compra habitual es el supermercado de la enseña analizada, seguido de Alcampo (21\%). En lo que respecta al Programa B, salvo la fuerte presencia de la enseña (70\%), las compras parecen realizarse de forma más dispersa, destacándose los establecimientos de Alcampo (7,7\%), Día (5\%) y El Corte Inglés $(4,3 \%)$, por delante de otras opciones minoritarias.

Con respecto a la frecuencia de compra, destacar que más de $2 / 3$ de la muestra ha comprado en la última semana (Tabla 4). Observamos también que el porcentaje de clientes del Programa B que ha comprado en la última semana, es inferior al del Programa A. Asimismo, el porcentaje de clientes que hace más de 1 mes que no compra es significativamente superior.

TABLA 4: Momento de la última compra en el supermercado de la enseña

\begin{tabular}{|l|c|c|c|}
\hline \multirow{2}{*}{ Momento compra } & Total muestra & \multicolumn{2}{c|}{ Programas áreas geográficas } \\
\cline { 3 - 4 } & $\%$ & A-Albacete (\%) & B-Barcelona (\%) \\
\hline En la última semana & 70,50 & 74,00 & 67,00 \\
\hline Entre 1 semana y 1 mes & 20,83 & 24,67 & 17,00 \\
\hline Hace más de 1 mes & 8,67 & 1,33 & 16,00 \\
\hline
\end{tabular}

Base: 600

Ji cuadrado con 2 grados de libertad $=42,5053 \quad(p=0,0000)$

Entre los motivos a los que aluden los clientes que no han comprado en la última semana destacan, en el área de Albacete "ningún motivo especial" (citado por un $24,4 \%$ ) y "trato del personal" (19,2\%). En el caso del programa de Barcelona, las razones que se aportan están más repartidas: "precios de los productos" $(21,4 \%)$, "por la variedad de los productos" (19,4\%), "cambio de domicilio" (18,4\%). Hay que destacar que la base en esta pregunta es 78 encuestados para el Programa A y 99 para el B. Las diferencias observadas entre las medias de las dos áreas son

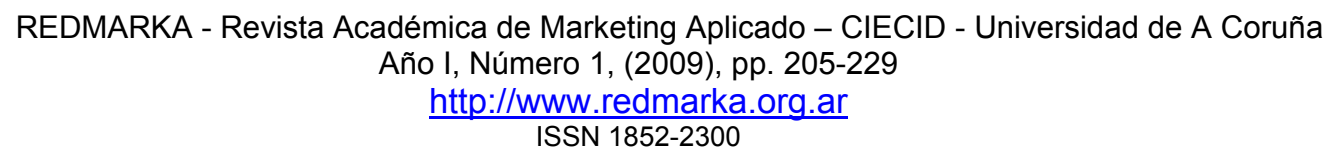


estadísticamente significativas a nivel 0,01 (Ji cuadrado con 9 grados de libertad = $60,3029 ; p=0,0000)$.

Atendiendo al importe de la compra del último mes en el supermercado, se observan también diferencias estadísticamente significativas, como muestra la Tabla 5.

TABLA 5: Importe de la compra del último mes en el supermercado de la enseña

\begin{tabular}{|l|c|c|c|}
\hline \multirow{2}{*}{ Importe } & Total muestra & \multicolumn{2}{c|}{ Programas áreas geográficas } \\
\cline { 3 - 4 } & $\%$ & A-Albacete (\%) & B-Barcelona (\%) \\
\hline Menos de $200 €$ & 27,33 & 32,00 & 22,67 \\
\hline 200 a $499 €$ & 31,50 & 35,67 & 27,33 \\
\hline 500 a $899 €$ & 21,67 & 19,33 & 24,00 \\
\hline 900 a $1499 €$ & 12,00 & 8,00 & 16,00 \\
\hline Más de $1500 €$ & 7,50 & 5,00 & 10,00 \\
\hline
\end{tabular}

En el Programa A cobran más peso las compras en los importes inferiores, mientras que en el Programa B destacan sobre la media la representatividad de los niveles superiores de gasto.

Si analizamos la relación entre la última compra realizada y la satisfacción con el supermercado y el programa de fidelización, no podemos concluir que los clientes más insatisfechos son los que hace más tiempo que no compran en el supermercado. Con respecto a la relación entre la última compra y la satisfacción con el programa de fidelización, parece que los clientes que declaran estar extremadamente satisfechos, tanto con el establecimiento como con el programa, han realizado su última compra hace menos de 1 mes. Las diferencias observadas no son estadísticamente significativas. En cualquier caso, tampoco podríamos inferir que los clientes dejen de comprar como consecuencia de su insatisfacción con el programa de fidelización. 
Con respecto al valor de las compras realizadas en el último mes, se ha buscado su relación con la satisfacción general con el supermercado y con el programa de fidelización. A este respecto, no podemos concluir que existe una relación directa entre el volumen de compra en el último mes y el nivel de satisfacción con el supermercado ni la satisfacción con el programa de fidelización, si bien las frecuencias conjuntas de ambas variables que presentan las correspondientes tablas de contingencia reflejan cierta asociación entre los clientes insatisfechos o neutros -tanto con el supermercado como con el programa- e importes inferiores de compra.

\subsection{Uso de la tarjeta}

Antes que nada, destacar que un $86 \%$ de los clientes declara utilizar la tarjeta del supermercado. Entre los motivos declarados para no utilizarla, destacan aspectos relativos al olvido (mencionado por un $53,2 \%$ ), la pérdida (34,2\%), seguidos, muy por detrás, por argumentos que denotan insatisfacción con el programa "no me ofrece suficiente" (7,6\% de los clientes).

Por su parte, a la pregunta "¿Tiene tarjeta de fidelización de algún otro supermercado?", responde afirmativamente $1 / 3$ de la muestra. En las respuestas a estas tres primeras cuestiones no se aprecian diferencias significativas entre los clientes de los dos programas analizados.

Con respecto al uso efectivo de las otras tarjetas de fidelización de otros supermercados, la respuesta a la pregunta "¿la utiliza cuando compra?" revela que su utilización está por debajo de la mitad de la muestra (46,8\% de los clientes), presentándose diferencias entre las dos áreas geográficas a nivel 0,05: el índice de uso de otras tarjetas es mayor para el Programa A $(55,2 \%)^{4}$.

Analizando la posible relación entre la satisfacción con el programa y el uso de la tarjeta del programa (a través de la tabla de contingencia que recoge las frecuencias conjuntas de las variables satisfacción general con el programa de fidelización y uso

\footnotetext{
${ }^{4} \mathrm{Ji}$ cuadrado con 1 grados de libertad $=5,2610 \quad(p=0,0218)$

REDMARKA - Revista Académica de Marketing Aplicado - CIECID - Universidad de A Coruña Año I, Número 1, (2009), pp. 205-229 http://www.redmarka.org.ar 
de la tarjera del programa) debemos destacar que niveles superiores de satisfacción ("bastante satisfecho", "muy satisfecho", "extremadamente satisfecho") aparecen asociados a un uso de la tarjeta del supermercado por encima de la media. No obstante, estadísticamente, debe rechazarse que las diferencias observadas sean significativas (Ji cuadrado con 9 grados de libertad = 8,7385; $p=0,4618$ ).

Por su parte, al estudiar relación entre la satisfacción con el programa y la tenencia y uso de otras tarjetas de fidelización de otros supermercados, se pone de manifiesto que clientes con niveles inferiores de satisfacción con el programa del supermercado ("Extremadamente insatisfecho" y "muy insatisfecho"), disponen de otra tarjeta alternativa por encima del resto de los grupos. Asimismo, se observa que la situación declarada de no uso de otras tarjetas aparece más asociada a los clientes pertenecientes a niveles superiores de satisfacción con el programa. Estas diferencias, no obstante, no se muestran estadísticamente significativas ( $p=0,3469$ у $p=0,2990$, respectivamente).

\section{Conclusiones}

- Los clientes están satisfechos con el programa de fidelización del supermercado tal como muestra la puntuación media de 7,51 que le otorgan. Atendiendo a la satisfacción con cada tipología de programa, se advierte que la puntuación en el programa de A está tres décimas por encima del B. Estas diferencias, aún muy sensibles, pueden apoyar la toma de decisiones.

- Buena capacidad de diferenciación de los elementos clave de cada programa, que sin embargo no resultan los más valorados.

- Escasa notoriedad de las acciones promocionales asociadas a los programas. Podemos concluir que no existe una notoriedad elevada en los envíos que realiza el supermercado, tanto de los que forman parte del plan de contactos anual, como de los últimos enviados. Éste se plantea entonces como uno de los aspectos más desfavorables en el programa. Parece como si la inversión y esfuerzo de la enseña por llegar a "impactar" a sus clientes no lograse su 
objetivo. Esto debe hacer meditar sobre la conveniencia de hacer menos envíos a lo largo del año y concentrarlos en momentos de alta implicación para el cliente (cumpleaños y Navidades, por ejemplo). Habría que analizar, no obstante, el impacto en las ventas de un envío determinado para confirmar estos resultados.

- No podemos concluir que exista dependencia entre el momento de la última compra o el importe de la compra del último mes y la satisfacción con el supermercado y el programa de fidelización, aunque se pone de manifiesto que los clientes que declaran estar extremadamente satisfechos, han realizado su última compra hace menos de 1 mes, y se refleja cierta asociación entre los clientes insatisfechos o neutros, tanto con el supermercado como con el programa, e importes inferiores de compra. Estos indicios pueden abrir una línea de investigación sobre el nivel de tolerancia de los clientes hasta que deciden el cambio de supermercado.

- Podemos afirmar que, al margen de las diferencias -más o menos relevantesque se derivan de las dos estrategias de recompensa analizadas, los factores que se muestran como verdaderamente relevantes en la satisfacción con el club de clientes del supermercado son la percepción del valor que ofrece la adscripción al programa de que se trate (sea mediante la compra directa, sea mediante el volumen de compras acumulado), el potencial que presenta la información de las transacciones en punto de venta para adaptar los incentivos a los hábitos de compra y, en última instancia, la satisfacción con el propio establecimiento (del que el programa constituye un extra y sin el cual esta herramienta de fidelización carece de entidad).

- Se pone de manifiesto la necesidad de reflexionar sobre la eficacia y la coherencia de la línea estratégica que sigue la compañía en términos de satisfacción de clientes:

- Binomio fidelización/satisfacción

- Seguimiento de niveles de satisfacción con la enseña: calidad de servicio percibida, frecuencia de compra, recomendación... 
- Vinculación a la promoción de elementos tangibles (elementos materiales unidos a la promoción) e intangibles (elementos diferenciadores en términos de comunicación).

\section{Referencias bibliográficas}

Alfaro, M. -Coord.- (2004): Temas Clave en Marketing Relacional, Barcelona: Gestión 2000.

AlCAide, J.C. (2006): "Cinco factores críticos para lograr la lealtad del cliente", Harvard Deusto Marketing \& Ventas, $n^{\circ} 76$, Septiembre/Octubre 2006, pgs. 30-36.

ALCAIDE, J.C. (2002): Alta fidelidad. Técnicas e ideas operativas para lograr la lealtad del cliente a través del servicio, Madrid: Esic.

Ayala TAyloR, G. (2001): "Coupon Response in Services", Journal of Retailing, Vol. 77, pgs. $139-151$.

Baumann, Ch.; Burton, S. y Elliott, G. (2005): "Determinants of Customer Loyalty and Share of Wallet in Retail Banking", Journal of Financial Services Marketing, Vol. 9, 3, pgs. 231-248.

Bennett, R. Y Rundle-Thiele, S. (2005): "The Brand Loyalty Life Cycle: Implications for Marketers", Brand Management, Vol. 12, 4, April, pgs. 250-263.

BUtSCHER, S.A. (1998): Customer Clubs and Loyalty Programmes. A Practical Guide, Hampshire; England: Gower Publishing Limited.

CAmpbelL, R. (2004): "The Development of the Largest Loyalty Brand in the UK: Nectar", European Retail Digest, Vol 41, Spring, pgs. 1-3 
CapizzI, M.T.y Ferguson, R. (2003): "Loyalty Trends For The 21st Century", Colloquy Talk, Paper 6.03, July, pgs. 1-15.

CORTINAS, M.; ElORZ, M.; MÚGICA, J.M. (2005): "Loyalty Cards, are Retailers Ignoring Non-Card-Holder Behaviour?", European Retail Digest, Vol 45, Spring, pgs. 18-20.

CRIÉ, D. Y MICHEAUX, A. (2006): "From Customers Data to Value: What is Lacking in the Information Chain?", Database Marketing \& Customers Strategy Management, Vol. 13, 4, pgs. 282-299.

CuthBERTSON, R. (1998): "Loyalty Cards Schemes in Retailing across Europe", European Retail Digest, Vol 20, December, pgs. 5-7.

DAVIES, G. (1998): "Loyalty Cards can Erode Loyalty. Only Customers Relationship Programmes can Build it", European Retail Digest, Vol 20, December, pgs. 9-13.

DICK, A.S. Y BASU, K. (1994): "Customer Loyalty: Towards an Integrated Conceptual Framework", Journal of Academy of Marketing Science, Vol. 22, 2, pgs. 99-113.

DuFFY, D.L. (1998), "Customer Loyalty Strategies", Journal of Consumer Marketing, Vol. 15, 5, pgs. 435-448.

GoldSMITH, R. E. (2004): "Current and Future Trends in Marketing and their Implications for the Discipline", Journal of Marketing, Fall, pgs. 10-17.

HALLBeRG, G. (2004): "Is Your Loyalty Programme Really Building Loyalty? Why Increasing Emotional Attachment, not Just Repeat Buying, is Key to Maximising Programme Success", Journal of Targeting, Measurement and analysis for Marketing, Vol. 12, 3, 2004, Pp. 231-241. 
HUETE, LUIS M. (1997): Servicios y Beneficios, Bilbao: Ediciones Deusto.

IYER, R.Y MUNCY, J.A. (2005): "The Role of Brand Parity in Developing Loyal Customers", Journal of Advertising Research, June 2005, Pp. 222-229.

Jones, T. O. y PASSER, W. E. (1995), "Why Satisfied Customers Defect", Harvard Business Review, November, pgs. 88-89

KIVETZ, R.; URMINSkY, O. Y Zheng, Y. (2006): "The Goal-Gradient Hypothesis Resurrected: Purchase Acceleration, Illusionary Goal Progress and Customer Retention", Journal of Marketing Research, Vol. 43, February, pgs. 39-58.

Martínez-Ribes, J.M.; De Borja L. y Carvajal, P. (1999): Fidelizando Clientes, Barcelona: Ediciones Gestión 2000.

Molina, A.; Martín-Consuegra, D. y Esteban A. (2006): "Marketing de Relaciones en la Distribución Comercial", Distribución y Consumo, Noviembre-Diciembre, pgs. 127-136.

O'DeLL, S. Y PAJUnEN, J.A. (1997): The Butterfly Customer, Canada: John Wiley And Sons, Canada Ltd.

ORTEGA, E. Y ReCIO, M. (1997): "Fidelización de Clientes y Marketing de Relaciones", Investigación y Marketing AEDEMO, nº 57, pgs. 33-40.

Parasuraman, A; Berry, L.L.y Zeitham, V.A. (1991): "Understanding Customer Expectations of Service", Sloan Management Review, Vol. 32, Spring, pgs. 39-48. 
RAHMAN, S.S. (2006): "Loyalty in Retail: a Strategic Success or a Management Failure", European Retail Digest, Winter 2005-2006, pgs. 57-60.

RAPHEL, N. Y RAYE, M. (1998): Loyalty Marketing Resource Book, New Jersey: Raphel Marketing.

ReICHHeLd, F. (1996): El Efecto Lealtad. Crecimiento, Beneficios y Valor último, Barcelona: Ariel Sociedad Económica.

ReINARES P.J. (2006): "Exploring the Benefits Obtained in a Loyalty Programme applied to Retailing", European Retail Digest, Vol 48, Winter 2005-2006, pgs. 48-52.

ReINARES P.J. Y PONZOA, J.M. (2002): Marketing Relacional. Madrid: Prentice Hall.

ReinaRes P.J.y ReinaRes, E. M. (2005): "Valoración Empírica de los Beneficios Obtenidos en un Programa de Fidelización aplicado al Comercio Minorista", Actas del XVII Encuentro de Profesores Universitarios de Marketing, Esic, Madrid, 22 y 23 de Septiembre, pgs. 219-236.

Rodríguez, Camarero y GutiérRez (2002): Lealtad y Valor en la Relación del consumidor. Una aplicación al caso de los Servicios Financieros, Actas del XIV Encuentros de Profesores Universitarios de Marketing, Esic, Granada, 18-20 de Septiembre, pgs. 429-443.

Roehm, M.L.; Bolman, E. Y Roehm, H.A. (2002): "Designing Loyalty-Building Programs for Packaged Good Brands", Journal of Marketing Research, Vol. 39, May, pgs. 202-213. 
RowLeY, J. (2004): "Loyalty and Rewards Schemes: How Much is Your Loyalty Worth?", The Marketing Review, 4, pgs. 121-138.

SANTESMASEs, M. (2005): Dyane Versión 3, Madrid: Ediciones Pirámide.

Setó, D. (2004): De la Calidad de Servicio a la Fidelidad del Cliente, Madrid: Esic.

SHANI, D. Y CHALASANI, S. (1992): "Exploiting Niches Using Relationship Marketing", Journal of Service Marketing, Vol. 6, pgs. 43-52.

Sirohi, N.; Mclaughlin, E.W. y Wittink, D.R. (1998): "A Model of Consumer Perceptions and Store Loyalty Intentions for a Supermarket Retailer", Journal of Retailing, Vol. 74, 2, pgs. 223-245.

Terblanche, N. S. y Boshoff, C. (2006): "The Relationship between a Satisfactory in-Store Shopping Experience and Retailer Loyalty", Journal of Business Management, 37, 2, March, pgs. 33-43

Too, L.H.; Souchon, A.L. Y THIRKelL, P.C. (2001): "Relationship Marketing and Customer Loyalty in a Retail Setting: A Dyadic Exploration", Journal of Marketing Management, Vol. 17, pgs. 287-319.

UNCLES, M. (1994): "Do you or your Customers a Royalty Scheme?", Journal of Targeting, Measurement and Analysis for Marketing, Vol. 13, pgs. 335 - 350

VAn Heerde, H.J.y BiJmolt, T.H. (2005): "Decomposing the Promotional Revenue Bump for Loyalty Program Members Versus Nonmembers", Journal of Marketing Research, Vol. 42, November, pgs. 443-457. 
VÁzquez, R.; Santos L.; Galguera L. Y Méndez M.P. (2000), Marketing de Relaciones: Estudio de las Tarjetas de Fidelización de las Compañías Aéreas, Universidad De Oviedo, Facultad De Ciencias Económicas.

WRIGHT, C. Y SPARKS, L. (1999): "Loyalty Saturation in Retailing: Exploring the End of Retail Loyalty Cards?", International Journal of Retail \& Distribution Management, Vol. 27, 10, 1999, pgs. 429-439.

\section{Para citar este artículo}

Labajo, Victoria - Tena, Antonio (25-02-2009). EFICACIA DE PROGRAMAS DE FIDELIZACIÓN EN SUPERMERCADOS. UN ENFOQUE COMPARATIVO DE DOS FORMAS DE CONSEGUIR LA LEALTAD DEL CLIENTE.

REDMARKA - CIECID - Unidad de Investigación en Marketing Aplicado-Universidad de A Coruña Año I, Número 1, V1, pp.205-229

ISSN 1852-2300

URL del Documento : http://www.cienciared.com.ar/ra/doc.php?n=1006 